\title{
Information transmission of 3.1 bits in absolute identification of auditory pitch
}

\author{
ANTE FULGOSI and BOZO ZAJA \\ Filozofski fakultet, 41000 Zagreb, Yugoslavia
}

Eleven tones (from 50 to $11,000 \mathrm{~Hz}$ ), differing only in pitch, were presented to five subjects 108 times each. The subjects were told that in the "training" phase of the experiment, they should reach a definite, but unspecified, "level" of performance before they could advance to the final or "test" phase of the experiment. In the final quarter of the experiment, the median value of the identification performance was 3.11 bits.

Experimental evidence about human ability to identify auditory pitch seems today inconclusive. Basically, there exists two kinds of data. Results of older investigations (Garner, 1962; Hartman, 1954; Miller, 1956; Pollack, 1952) show that, in the situation of absolute judgments of tones differing only in pitch, only 2.3 bits or five tones can be identified. This value is treated as the "channel capacity" or the upper limit of this ability.

On the other side, some more recent investigations have shown that there exists a practice effect when subjects are trained in identification of tones (Cuddy, 1968, 1970, 1972; Deutch, 1972; Heller \& Auerbach, 1972). Siegel (1972) and Siegel and Siegel (1972) have shown that the process of forgetting the information about pitch is extremely rapid in situations of absolute judgments and concluded that the concept of a fixed upper limit in absolute judgment of pitch is incorrect.

Results of Cuddy's (1968, 1970, 1972), Heller and Auerbach's (1972), as well as Siegel's (1972), experiments, while showing that some improvement in identification performance of pure tones differing only in pitch is possible, are still within the value of "channel capacity." This means that subjects in these experiments have not achieved the upper "limit" of transmission. For this reason, it is not clear if training is possible only below this limit (in which case such improvement could be expected) or if it is possible that training of absolute identification could bring the identification performance above the value of 2.3 bits. While it seems that the improvement below the value of 2.3 bits is quite well demonstrated, there is nothing that shows that such improvement can exceed this value. On the contrary, in the context of data showing the rapid forgetting and poor recognition of pure tones, such an improvement seems quite improbable. The present investigation tries to clear some of these dilemmas.

\section{METHOD}

\section{Subjects}

The subjects in this experiment were five undergraduate students of psy chology. Four of them were without any previous musical training. One studied music for several years. All had normal hearing.

\section{Stimuli}

Eleven tones of the following frequencies (in hertz) were used as stimuli: $50,150,300,550,1,000,1,700,2,600,4,000,5,600$, $8,000,11,000$. Duration of each stimulus was $5 \mathrm{sec}$. Interstimulus interval was $7 \mathrm{sec}$.

\section{Apparatus}

Tones were generated by a Bruel and Kjaer beat frequency oscillator Type 1022. This instrument was connected through a power amplifier with five pairs of Koos earphones (6 ohms) and to an artificial ear.

The loudness of all tones were $75 \mathrm{~dB}$ SPL. Loudness of all tones was held constant throughout the experiment.

Connected to this circuit was a timer which controlled duration of stimuli and the interstimulus interval.

\section{Procedure}

Since some recent investigations have shown that in absolute judgments experiments it is important to control the motivation of subjects (Chapanis \& Overbey, 1971), subjects in the present experiment were told that the experiment has two "phases": the training and the test phases. The training "phase" will last until the subjects reach a predetermined "level" of performance in the identification of tones after which they will have a short testing "phase." In fact, such phases did not exist. Beside this, the subjects were told to make a sign near the response identification which was correct. At the end of each session, the subjects were required to count these signs and to write the number in a corner of their protocol. The experimenter read loudly this number from each protocol so that each subject in the group was informed about the results of others and aware that the experimenter knew his. After that, the usual instructions for the absolute identification procedure was given.

Before the beginning of the experiment, all tones were presented from the lowest to the highest, and the experimenter identified each by the proper number (from 1 to 11 ). This was done also before the beginning of each session. After that, nine random order presentations of all 11 tones were given for the purpose of practice.

There were six experimental sessions, one each day. In each session, there were 18 presentations of each tone in random order, or 198 presentations in all. Random order of presentation of stimuli was determined for each 99 presentations.

After each presentation and after the subjects wrote their response, the experimenter pronounced the right identification number for that stimulus.

The informational value of this set of stimuli was 3.46 bits. 


\section{RESULTS}

Since in an earlier investigation (Fulgosi \& Bartolovic, 1971) it was shown that the amount of transmitted information (T) calculated from the whole range of the transmission experiment is not representative for the final level of identification performance and cannot be interpreted as an index of channel capacity, $T$ values in the present experiment were calculated for each quarter of the experiment (for successive 297 presentations or for successive 27 presentations of each stimulus). T values were calculated for each subject separately. Results of these analyses appear in Table 1.

Values of $\mathrm{T}$ for the whole range of the experiment appear in the bottom line of Table 1 .

Table 1

Amount of Information (in Bits) Transmitted in Different Phases of the Experiment

\begin{tabular}{rrrrcccc}
\hline \multicolumn{2}{l}{$\begin{array}{l}\text { Serial No. of } \\
\text { Presentations } \\
\text { from }\end{array}$} & \multicolumn{6}{c}{ Subjects } \\
\cline { 2 - 7 } & \multicolumn{1}{c}{ to } & $1^{*}$ & 2 & 3 & 4 & 5 \\
\hline 1 & 297 & 2.71 & 2.38 & 2.46 & 2.18 & 2.11 \\
298 & 594 & 3.09 & 2.71 & 2.95 & 2.42 & 2.52 \\
595 & 891 & 3.31 & 2.99 & 3.00 & 2.74 & 2.61 \\
892 & 1188 & 3.40 & 3.13 & 3.03 & 3.11 & 2.79 \\
1 & 1188 & 3.06 & 2.74 & 2.73 & 2.53 & 2.39 \\
\hline
\end{tabular}

Note. Italicized numbers are median values.

*Subject with several years of music school.

\section{DISCUSSION}

Results of the present experiment indicate that subjects are able to transmit more information about the pitch than was shown in earlier experiments and that the value of 2.3 bits does not represent the upper limit of this ability. Only at the beginning of the experiment was median value of transmission near the traditional "channel capacity." In the last quarter of the experiment, median value of transmission rose to 3.11 bits which corresponds to nine categories (almost double the number of categories than earlier) and equal to the number of categories found for tones differing simultaneously in pitch and loudness (Pollack, 1953).

The experiment also shows that it is possible to train adequately motivated subjects to identify pure tones differing only in pitch but that such training is quite slow. It seems that at the end of the experiment, two subjects were still progressing. These results show that rapid forgetting of information about pitch can be counterbalanced by training. The subject who had several years of musical schooling achieved, in the last quarter of the experiment, transmission of 3.4 bits, which is almost perfect transmission of these 11 stimuli.

\section{REFERENCES}

Chapanis. A.. \& Overbey, C. M. Absolute judgments of colors using natural color names. Perception \& Psychophysics, 1971 , 9. $356-360$.

Cuddy, L. L. Practice effects in the absolute judgment of pitch. Journal of the Acoustical Society of America, 1968, 43. 1069-1076.

Cuddy, L. L. Training the absolute judgment of pitch. Perception \& Psychophysics. 1970. 8, 265-269.

Cuddy, L. L. Comments on "Practice effects in the absolute judgment of frequency" by Heller and Auerbach. Psychonomics Science, 1972. 28. 68.

Deutch. D. Octave generalization and tone recognition. Perception \& Psychophysics. 1972, 11, 411-412.

DEUTCH, D. Interference in memory between tones adjacent in the musical scale. Journal of Experimental Psychology, 1973, 100, 228-231.

Fulgosi, A., \& Bartolovíc, B. Practice and some methodological problems in unidimensional information transmission. Perception \& Psychophysics, 1971, 10. 283-285.

GARNER, W. R. Uncertainty and structure as psychological concepts. New York: Wiley, 1962.

HARTMAN. E. B. The influence of practice and pitch distance between tones on the absolute identification of pitch. American Journal of Psychology. 1954, 67, 1-14.

Heller, M. A.. \& Auerbach, C. Practice effects in the absolute judgment of frequency. Psychonomic Science, 1972, 26. 222-224.

Miller. G. A. The magical number seven plus or minus two. Psychological Review, 1956, 63, 81-97.

Pollack, I. The information of elementary auditory displays. Journal of the Acoustical Society of America, 1952, 24, 745.749.

Pollack, I. The information of elementary auditory displays, II. Journal of the Acoustical Society of America, 1953, 25, 765-769.

Siegel. W. Memory effects in the method of absolute judgment. Journal of Experimental Psychology, 1972. 94, 121-131.

Siegel, J. A.. \& Siegel, W. Absolute judgment and paired associate learning: Kissing cousins or identical twins, Psychological Review, 1972, 79, 300-316.

(Received for publication June 27, 1975.) 